\title{
Spectral line shape modeling and ion temperature fluctuations in tokamak edge plasmas
}

\author{
Y. Marandet ${ }^{1}$ and J. W. Dufty ${ }^{1}$ \\ ${ }^{1}$ Department of Physics, University of Florida, P.O. Box 118440, Gainesville, Florida 32611-8440,USA
}

\begin{abstract}
In this work, we use a passive advection model for ion temperature fluctuations, in order to investigate their effects on Doppler Spectral line shapes. The relevance of the model is discussed in the framework of the Braginskii equations, and the subsequent Probability Density Function evaluation relies on results obtained in neutral fluids. The resulting Doppler line profiles are shown to exhibit characteristic exponential tails.
\end{abstract}

PACS numbers: 32.70.Jz, 52.35.Ra

\section{INTRODUCTION}

The understanding of turbulence in fusion devices has made great progress since it was first realized that turbulent fluctuations could explain the anomalously high level of cross magnetic field transport which was plaguing experiments. Theoretical studies, many of which have focused on the identification of underlying linear or non linear instabilities [1], have produced valuable results. Direct Numerical Simulation, which is the only tool able to encompass the complexity of the problem (non linear equations, multiple fields and complex geometry), has shed additional light on the respective contributions of these instabilities (e.g. 2]). Edge plasma turbulence has specific properties and is, in particular, characterized by high fluctuation levels which can rise up to $30 \%$, 4, 4]. The development of sophisticated diagnostics methods now provides detailed experimental data on density fluctuations (e. g. [5]), which impose more stringent constraints on the theory. However, very few measurements of ion temperature fluctuations have been reported to date. A notable exception is Ref. [4], in which High Frequency Charge Exchange Recombination Spectroscopy is used to show that ion temperature fluctuations rates can be large. In previous works [6, 7], we have investigated the possible role of turbulence on Doppler line shapes obtained by passive spectroscopy. Ion temperature fluctuations were shown to lead to significant modifications in the line wings, provided their PDF (Probability Density Function) had fat tails. This suggests that passive spectroscopy, for which the experimental set-up is relatively simple, might provide information on ion temperature fluctuations. Here, making use of results obtained in neutral fluids, we show that even a very simplified model for ion temperature fluctuations, i.e. passive advection, leads to non Gaussian PDFs. The corresponding asymptotic behavior of the Doppler line profiles is obtained analytically.

\section{DOPPLER SPECTRA IN TURBULENT PLASMAS}

The spectra $I_{m}(\Delta \lambda)$ measured using passive spectroscopy is obtained from integration both during the acquisition time of the spectrometer $\tau$ and along the line of sight (LOS) (Oz). The corresponding expression is

$$
I_{m}(\Delta \lambda)=\frac{1}{\tau} \int_{0}^{\tau} d t \frac{1}{L} \int d z B(z, t) I(\Delta \lambda, z, t),
$$

where $L$ is the emitting zone length, $B(z, t)$ the brightness of the line and $I(\Delta \lambda, z, t)$ the local line shape. Both of these quantities are determined by the plasma parameters and the line under study. Here we consider $D_{\alpha}$ ( $n=3$ to $n=2$ transition, $\lambda_{0}=6561 \AA$ ), which is intense and routinely monitored. The brightness essentially depends on the electron density and temperature. For ionizing edge plasma conditions, that is $N_{e}=10^{19}-10^{20} \mathrm{~m}^{-3}$ and $T_{e}=10-100 \mathrm{eV}$, Stark effects can be safely neglected. We will therefore deal with the Doppler line profile of one Zeeman component. Here we only consider the contribution from the class of neutrals created locally by charge exchange, $I(\Delta \lambda, z, t) \rightarrow$ $I^{c x}(\Delta \lambda, \mathbf{X})$. In fact, neutrals created via other channels, i.e molecular dissociation and charge exchange in the inner plasma are not strongly coupled to the ions at the edge [8], i.e. to edge turbulence. These neutrals can be accounted for using neutral transport codes. The charge exchange neutrals Velocity Distribution Function (VDF) along the LOS, $f^{c x}(v, \mathbf{X})$, is related to the Doppler profile by $f^{c x}(v, \mathbf{X}) d v=I^{c x}(\Delta \lambda, \mathbf{X}) d \Delta \lambda$. Here it has been made explicit that the space and time dependence of the spectra and VDF occurs only through the local ion fluid fields $\mathbf{X}(z, t)$ (local number and charge densities, species temperatures, and flow velocities), since the ion VDF is assumed to be a local Maxwellian. We consider the case where the LOS is such that the average gradients of these fields along its 
direction (e.g., parallel to the magnetic field lines) are weak. It was shown in Ref. [6, 7] that the measured spectra can then be written as

$$
I_{m}^{c x}(\Delta \lambda)=\int d \mathbf{X} W(\mathbf{X}) B(\mathbf{X}) I^{c x}(\Delta \lambda, \mathbf{X})
$$

where $W(\mathbf{X})$ is the joint PDF of the fields. To proceed, the role of the different fluid fields can be investigated separately. For Doppler line shapes, fluid velocity and temperature fluctuations give rise to the main effects, because the shape of the local VDF $f_{c x}$ does not depend on $N_{e}$. We further simplify the problem by considering ion temperature fluctuations alone. The rationale behind that assumption is twofold : first, the role of velocity fluctuations is well known in plasma spectroscopy [9]. Secondly, the analysis carried out in our previous work showed that line wings are significantly affected by temperature fluctuations characterized by a slowly decaying tail. Therefore, we expect that inclusion of velocity fluctuations would not significatively shroud the conclusions drawn here, since line wings could only be affected by fluid velocities several times larger than the ion thermal velocity. Moreover, if the LOS is parallel to the magnetic fields, there are conditions under which parallel velocity fluctuations are small while temperature fluctuations are not [4]. As a result, the remainder of this contribution will be devoted to temperature fluctuations.

\section{SIMPLIFIED MODEL FOR THE ION TEMPERATURE}

We start from the Braginskii equations [10], and focus on the ion thermal balance

$$
\frac{3}{2} d_{t}\left(n_{i} T_{i}\right)+\frac{5}{2} n_{i} T_{i} \nabla \cdot \mathbf{v}_{i}+\pi:: \sigma+\nabla \cdot \mathbf{q}_{i}=Q_{i}\left(T_{i}, \mathbf{v}\right)
$$

which we simplify using the drift wave ordering. Here $d_{t}=\partial_{t}+\mathbf{v}_{i} \cdot \nabla$ stands for the convective derivative, $\mathbf{q}_{i}$ for the thermal flux, $\pi_{\alpha \beta}$ is the stress tensor, $\sigma_{\alpha \beta}=\partial_{\alpha} v_{\beta}$ and $Q_{i}$ is the energy exchange with other species. We consider fluctuations having a small scale $\tilde{l}$ with respect to the quasi equilibrium background of radial gradient length $L_{\perp}$. Typically $\rho_{s} \lesssim \tilde{l}$, where $\rho_{s}$ is the ion Larmor radius calculated with the electron temperature. For each field $X$, we distinguish between its time averaged part $\bar{X}$ and its fluctuating part $\tilde{X}$. The small scale parameter with respect to which the fluid equations are expended is $\delta=\tilde{l} / L_{\perp}$. The perpendicular velocity is given by $\mathbf{v}_{i \perp}=\mathbf{v}_{E}+\mathbf{v}_{i}^{\star}+\mathbf{v}_{i}^{p}$, that is by the sum of the electric drift, ion diamagnetic and ion polarization velocities. The diamagnetic velocity does not directly advect temperature, because for low $\beta$ (the ratio of the kinetic to the magnetic pressure) its effect cancels out with that of the diamagnetic thermal flux, up to magnetic curvature terms which will be neglected in the following. The same approximations ensure that $\nabla \cdot \widetilde{\mathbf{v}}_{E} \simeq 0$, i.e. the fluctuating electric drift velocity flow is incompressible. The ion polarization velocity is of higher order in $\delta$ and we further neglect parallel advection, which is justified in the drift ordering 11]. The resulting equation for ion temperature fluctuations is then

$$
\left[\partial_{t}+\left(\overline{\mathbf{v}}_{E}+\widetilde{\mathbf{v}}_{E}\right) \cdot \nabla-\kappa_{i \perp} \nabla^{2}\right] \widetilde{T}_{i}=\widetilde{S}(\mathbf{r}, t)
$$

where $\kappa_{i \perp}$ is the ion perpendicular thermal diffusivity, and all the terms on the r.h.s. have been lumped into a source, that is $\widetilde{S}(\mathbf{r}, t)=\widetilde{\mathbf{v}}_{E} \cdot \nabla \bar{T}_{i}-\overline{\widetilde{\mathbf{v}}}_{E} \cdot \nabla \widetilde{T}_{i}+\widetilde{Q}_{i}$. The energy exchanges between electrons and ions $Q_{i e}$ and ions and neutrals $Q_{i n}$ are neglected on the turbulent time scales, i.e. $\widetilde{Q}_{i}=Q_{i}-\bar{Q}_{i} \simeq 0$. Also, the second term is zero by homogeneity, so that $\widetilde{S}(\mathbf{r}, t)=\widetilde{\mathbf{v}}_{E} \cdot \nabla \bar{T}_{i}$. The mean temperature gradient therefore naturally provides a source term for the fluctuations through its coupling to $\widetilde{\mathbf{v}}_{E}$. Since the scale of the turbulent fluctuations is such that $\tilde{l} \ll L_{\perp}$, in the following the gradient will be assumed to be constant and directed along $x$, namely $\nabla_{x} \bar{T}_{i}=g$. Advection by the mean flow $\overline{\mathbf{v}}_{E}$ in Eq. (4) can be formally removed by introducing suitable Lagrangian coordinates. This does not interfere with the following developments, so that we will assume $\overline{\mathbf{v}}_{E}=0$. Rigorously speaking, the evolution of $\widetilde{\mathbf{v}}_{E}$ is not independent from that of $\widetilde{T}_{i}$ (see for example the system of equations considered in Ref. [2]). However, the ion temperature does not play any role in the parallel electron dynamics, in contrast to the electron temperature which is strongly coupled to the electrostatic potential, and hence to $\widetilde{\mathbf{v}}_{E}$. In addition, many if not most edge turbulence studies carried out so far did not include ion temperature fluctuations, by considering cold ions $\left(T_{i} \ll T_{e}\right)$. This approximation is justified partly by the fact that ion temperature does not play a fundamental role for the instabilities which are thought to be dominant in edge plasmas (collisional drift waves, resistive ballooning). In fact, inclusion of $\widetilde{T}_{i}$ does not qualitatively change the nature of the turbulence observed in the simulations [2], and the few experimental results available are not inconsistent with passive advection, as pointed out in [4]. In this work, we therefore treat ion temperature as 
a passive scalar driven by the fluctuating electric drift velocity field. This has the major advantage to lead to an analytically tractable model. Let us now specify the typical values of the Péclet and the Prandtl numbers, which will play a role in the next section, for edge plasma turbulence. The Péclet number, $\mathrm{Pe}=\tilde{l} \tilde{v} / \kappa_{i \perp}$, where $\tilde{l}$ and $\tilde{v}$ are the typical length scales and velocity of the turbulent fluctuations, controls the relative importance of advection and diffusion. If we take $\tilde{l} \sim 10 \rho_{s} \sim 2 \times 10^{-3} \mathrm{~m}$ and $\tilde{v} \sim \tilde{v}_{E} \sim \tilde{v}_{i}^{\star} \sim T_{i} /\left(e B L_{\perp}\right) \sim 75 \mathrm{~m} . \mathrm{s}^{-1}$ with $L_{\perp}=2 \mathrm{~cm}$, we get $\mathrm{Pe} \sim 10^{2} \gg 1$, so that advection dominates the heat transport. The Prandtl number $\operatorname{Pr}=\nu_{i \perp} / \kappa_{i \perp}$, where $\nu_{i \perp}$ is the perpendicular ion viscosity is of order unity since $\nu_{i \perp} / \kappa_{i \perp} \sim 1[10]$.

\section{REVIEW OF ANALYTICAL CALCULATIONS OF THE PASSIVE SCALAR PDF}

Since $\tilde{T}_{i}$ and $\tilde{\mathbf{v}}$ are rapidly fluctuating quantities, we adopt a stochastic description of the velocity field. For the sake of tractability, the latter is described by a Gaussian probability density functional, whose correlation function is chosen so as to reproduce some of the main features of turbulence [12], that is

$$
C_{\alpha \beta}(\mathbf{r}, t)=\left\langle v_{\alpha}(\mathbf{r}, t) v_{\beta}(0,0)\right\rangle=\Pi_{\alpha \beta} V \xi \exp (-|\mathbf{r}| / \xi) \delta(t)
$$

where $\Pi_{\alpha \beta}(\mathbf{r})$ ensures consistency with incompressibility [13] (at zeroth order in $\mathbf{r}, \Pi_{a b}=\delta_{a b}$ ), and the white noise limit has been taken. Here, the correlation length $\xi$ and the typical velocity $V$ are such that $\xi \sim \tilde{l}$ and $V \sim \tilde{v}$. The time average is thus replaced by an ensemble average denoted by $\langle\cdot\rangle$. In this section, we show how the ion temperature PDF $W(\theta)=\left\langle\delta\left(\theta-\widetilde{T}_{i}(\mathbf{r}, t)\right)\right\rangle$, or equivalently its Fourier transform $Z(\lambda)=\left\langle\exp \left(-i \lambda \widetilde{T}_{i}(\mathbf{r}, t)\right)\right\rangle$, can be obtained from Eq. (41). Here, $\theta$ is a sample space variable, and $\lambda$ its conjugated Fourier variable. Mathematically, the brackets denote functional integration over the field $\widetilde{\mathbf{v}}$ at all space and time points. The PDF does not depend on $t$ and $\mathbf{r}$ because of stationarity and homogeneity, the latter stemming from the fact that the average temperature gradient is constant. The derivation presented here is drawn from the neutral fluid community, which has given much attention to the passive advection problem. Indeed, its study allows to unravel important effects also at play in Navier-Stokes turbulence. The passive scalar equation is linear in $\widetilde{T}_{i}$, but bilinear in the fluctuations and can thus generate non linear effects. The scalar PDF $W(\theta)$ can therefore have strong non Gaussian features even though the velocity field is Gaussian. This is very well illustrated by the early work of Sinai and Yakhot, who found power law tails for the PDF in homogeneous decaying turbulence 14]. The derivation relies on a transport equation for the PDF, which involves coefficients whose temperature dependence has to be determined from rather uncontrolled assumptions. This difficulty arises because diffusion introduces correlations between the scalar field and its gradients. The physical reason for this is most easily understood using a Lagrangian picture. In the weak thermal diffusion limit (i.e. large Péclet number $\mathrm{Pe} \gg 1$, see below), advection is the dominant initial effect and the fluid particles follow the Lagrangian trajectories defined by $d_{t} \mathbf{R}(t)=\mathbf{v}\left(\mathbf{R}(t), t \mid \mathbf{R}_{\mathbf{0}}\right)$ where $\mathbf{R}(t=0)=\mathbf{R}_{0}=\mathbf{r}$. The velocity field is linearized around the Lagrangian trajectory $\left(\widetilde{v}_{\alpha}(\mathbf{r}, t) \rightarrow \widetilde{v}_{\alpha}(\mathbf{R}(t), t)+\sigma_{\alpha \beta}(\mathbf{R}(t), t)\left(r_{\beta}-R_{\beta}(t)\right)\right)$, where $\sigma_{\alpha \beta}(\mathbf{R}(t), t)=\partial_{\alpha} v_{\beta}(\mathbf{R}(t), t)$ is the strain field along the trajectory. Two points in a fluid element, initially close will be displaced by this strain field. Due to the condition of incompressibility, one direction will involve an exponentially large separation as a function of time. As a consequence of this so-called "advective stretching", the spectra of temperature fluctuations are extended to smaller and smaller scales. When the diffusive scale is eventually reached, regions of large gradient are selectively dissipated, introducing correlations between the temperature field and its gradient [15. The PDF obtained in Ref. 14] has been used as an example in our previous work [, , 7], but from the section 3 it is clear that an additive noise should also be taken into account. The corresponding transport equation for the PDF could straightforwardly be written down, but finding its solution would require further assumptions on the correlations between $\widetilde{T}_{i}$ and $\widetilde{S}[16]$. From the discussion above it is obvious that a Lagrangian description would allow to capture more of the physics. The remainder of this section will be devoted to show how this unfolds. The first difficulty when carrying out the average in $Z(\lambda)$ lies in the fact that there are two different sources of noise associated with $\widetilde{\mathbf{v}}_{E}$, one additive $\left(\widetilde{S}_{g} g \widetilde{v}_{E x}\right)$ and one multiplicative $\left(\widetilde{\mathbf{v}}_{E} \cdot \nabla\right)$. An elegant way to disentangle their respective contributions is to calculate the Green's function $G$ for Eq. (4), satisfying

$$
\widetilde{T}_{i}(\mathbf{r}, t)=\int d \mathbf{r}^{\prime} d t^{\prime} G\left(\mathbf{r}, t \mid \mathbf{r}^{\prime}, t^{\prime}\right) g \widetilde{v}_{E x}\left(\mathbf{r}^{\prime}, t^{\prime}\right)
$$

This has the advantage to allow averaging on the additive noise as a first step. The latter is independent of the statistics of $\widetilde{\mathbf{v}}_{E}$ in $G\left(\mathbf{r}, t \mid \mathbf{r}^{\prime}, t^{\prime}\right)$ in the limit of white noise. Physically speaking this occurs when the time scale for velocity field correlations is short compared to other relevant time scales for $\widetilde{T}_{i}$. In the following, all results quoted 
are in this limit. The resulting expression for the characteristic function is

$$
Z(\lambda)=\left\langle\exp \left(-\lambda^{2} \int_{-\infty}^{0} d t \int d \mathbf{k} \widehat{D}(\mathbf{k})|\widehat{G}(\mathbf{k}, t)|^{2}\right)\right\rangle_{M}
$$

where $\langle\cdot\rangle_{M}$ stand for the ensemble average over the multiplicative noise, i.e. the $\mathbf{v}$ dependance of the Green function. $\widehat{D}(\mathbf{k})$ is the Fourier transform of the additive noise correlation function $D(\mathbf{r})=\langle\widetilde{S}(0, t) \widetilde{S}(\mathbf{r}, t)\rangle_{A}=g^{2} C_{x x}(\mathbf{r})$. This approach was first carried out in Ref. [12] using a path integral formulation to calculate the Green's function, assuming the above linearization of the velocity field around the Lagrangian trajectory. Strictly speaking, the latter is valid for $\operatorname{Pr} \gg 1$, but is thought to be also relevant to the cases where $\operatorname{Pr} \sim 1$ [12]. The same approximation allows to expand $C_{x x}(\mathbf{r})$ in terms of $|\mathbf{r}| / \xi$. The Green's function, Fourier transformed with respect to the starting point, is then found to be

$$
\widehat{G}\left(0,0 \mid \mathbf{k}_{0}, t\right)=e^{i \mathbf{k}_{0} \cdot \mathbf{r}(t ; 0,0)} \exp \left(-\frac{\kappa_{i \perp}}{2} \int_{t}^{0} d t^{\prime} \mathbf{k}^{2}\left(t^{\prime}\right)\right),
$$

where the wave number $\mathbf{k}(t)$ is defined by the time ordered product $(\mathcal{T}$ stands for latest times on left)

$$
k_{\alpha}(t)=\mathcal{T}\left[\exp \left(-\int_{t}^{0} \sigma\left(t^{\prime}\right) d t^{\prime}\right)\right]_{\alpha \beta} k_{0 \beta}
$$

Here $\mathbf{r}(t ; 0,0)$ is the backwards Lagrangian trajectory, i.e. the position at which the fluid particle which is at time $t=0$ at position $\mathbf{r}=0$ was at time $t<0$, not taking into account diffusion. The second term in Eq. (8) is a damping term originating from diffusion, where the time dependence of $\mathbf{k}(t)$ describes the effect of stretching integrated along the Lagrangian trajectory. The norm of $\mathbf{k}$ grows exponentially for the velocity field considered here, so that this second term basically behaves as a step function at a time $t^{\star}$, which can be defined as $\left|\widehat{G}\left(0,0 \mid \mathbf{k}_{0}, t^{\star}\right)\right| \sim 1 / 2$. The larger the strain along the trajectory, the smaller $t^{\star}$. For $t<t^{\star}$ the fluid particle follow the diffusionless Lagrangian trajectory with constant temperature, and then the latter is quickly homogenized because of the large gradients induced by stretching. Since the modulus of the Green's function only depends on the velocity field through the strain tensor $\sigma$ (see Eq. (8)-(9)), the velocity average is then converted into an average over realizations of $\sigma$. Carrying out the calculation is tedious, but only involves some additional mild approximations [12]. The resulting PDF has a Gaussian core and an asymptotical behavior which is exponential, that is using non dimensional quantities

$$
W(\theta) \sim \frac{1}{|\theta|^{1 / 2}} e^{-\gamma|\theta|}
$$

where $\gamma=(d+6) / 2 d$ is the secular growth rate, and $d$ the dimensionality of the problem $(\gamma=2$ in 2D) 12 . The same result has been obtained using Jensen's field theoretical formalism 17]. Taking an additive noise into account, as required by the derivation of Eq. (4), therefore leads to a PDF which has an exponential tail instead of the power law tail obtained for decaying turbulence. The PDF is however still markedly non Gaussian.

\section{CALCULATIONS OF DOPPLER SPECTRA}

We now consider the modifications resulting from temperature fluctuations on line shapes, given the PDF obtained in the previous section. The Doppler line shape $I_{m}(\Delta \lambda)$ can be calculated numerically from Eq. (2) for any given $W(\theta)$, noting that the brightness of the line $B$ does not sensitively depend on the ion temperature. However, it is interesting first to obtain an analytical expression of the profile. This can be done for PDFs behaving asymptotically as $W(\theta) \sim \theta^{-\alpha} \exp -\left(\theta / \theta_{0}\right)^{\beta}$. The asymptotic behavior of the measured Doppler profile can then be calculated using a saddle point approximation, which leads to

$$
I_{m}(\Delta \lambda) \propto \frac{1}{|\Delta \lambda|^{\frac{\beta+2 \alpha-1}{\beta+1}}} \exp \left(-C\left(\theta_{0}, \beta\right)|\Delta \lambda|^{\frac{2 \beta}{\beta+1}}\right)
$$


where $C\left(\theta_{0}, \beta\right)=(\beta+1) /\left(\beta \zeta \theta_{0}\right)^{\beta / \beta+1}$, with $\zeta=\lambda_{0}^{2} /\left(c^{2} m_{i}\right), m_{i}$ being the ion mass and $c$ the speed of light. For $\beta=0$, the measured profile decays algebraically in accordance with Ref. [6, 7], while for an exponential PDF $(\beta=1)$, its decay is exponential too. This implies that ion temperature fluctuations characterized by an exponential PDF would lead to a conspicuous behavior in the line wings. As an example, we consider the following temperature PDF (adapted from Eq. (4.10) in Ref. [12]), having a Gaussian core and exponential tails

$$
W(\theta)=\frac{N}{(g \xi) \sqrt{g \xi(\mathcal{K} / V \xi) \ln \mathrm{Pe}}+|\theta|^{3 / 2}}\left(\theta+T_{0}\right) \exp \left(-\frac{2}{g \xi} \frac{\theta^{2}}{g \xi(2 \mathcal{K} / V \xi) \ln \mathrm{Pe}+\sqrt{2 \mathcal{K} / V \xi}|\theta|}\right)
$$

where $N$ is a normalization constant, $\xi$ and $V$ are defined by Eq. (5), and $\mathcal{K}=\int_{0}^{+\infty} C_{x x}(\mathbf{R}(t), t) d t$ is the eddy diffusivity, where $\mathbf{R}(0)=0$. The effective Peclet number $V \xi / \mathcal{K}$ is such that $\mathcal{K} / V \xi=1$ according to Eq. (5). This VDF is plotted on Fig. Th for $T_{0}=50 \mathrm{eV}, \mathrm{Pe}=10^{2}$, and two gradient values $g \xi=10 \mathrm{eV}$ and $15 \mathrm{eV}$. The resulting Doppler profiles are plotted on Fig. 1 $\mathrm{b}$, together with the Gaussian at $50 \mathrm{eV}$ corresponding to the fluctuation free case. The center of the line is not strongly affected, whereas significant deviations occur in the line wings. The

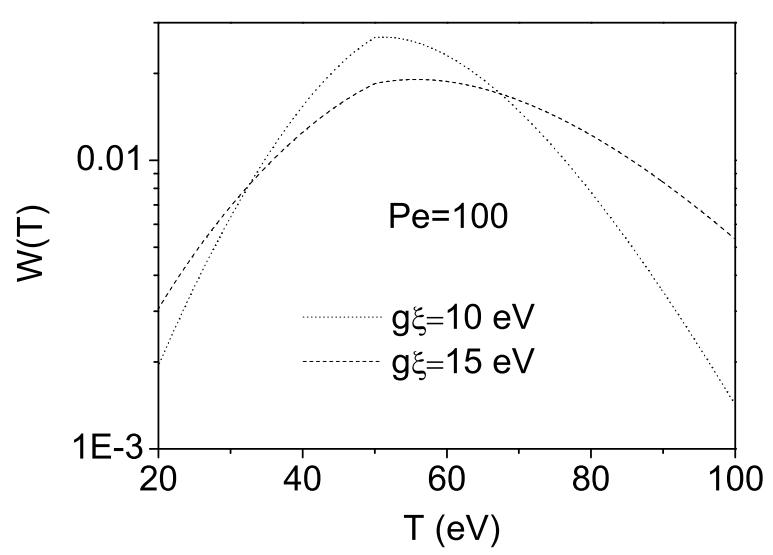

a)

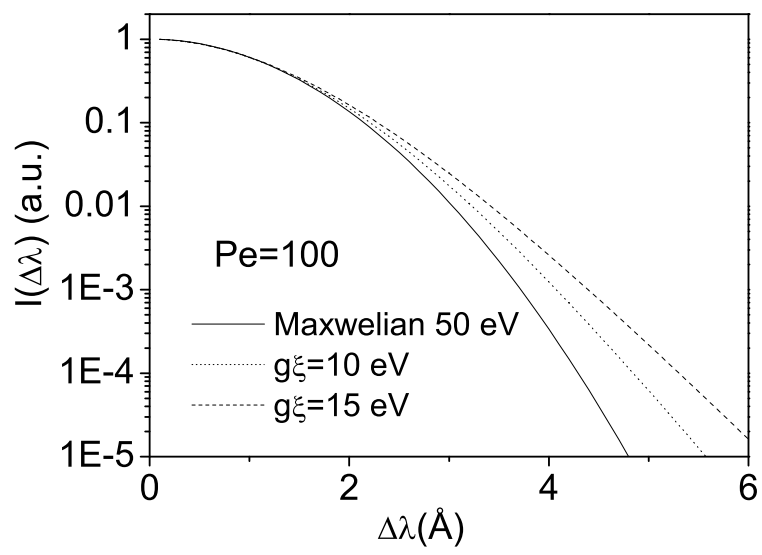

b)

FIG. 1: a) Plot of the PDF of Eq. (12) for $T_{0}=50 \mathrm{eV}, g \xi=10 \mathrm{eV}, \mathrm{Pe}=10^{2}, V \xi / \mathcal{K}=1$ (dotted line), and $g \xi=15 \mathrm{eV}$ (dashed line) . b) Plot of the resulting Doppler profile on a logarithmic scale (solid and dashed lines), showing the asymptotic exponential behavior. The solid line corresponds to the Gaussian Doppler profile obtained for $50 \mathrm{eV}$.

linear dependance characterizing an exponential fall off in log-linear scale is clearly seen. These results show that Doppler line profiles would indeed be affected by ion temperature fluctuations stemming from passive advection. Comparison to experimental spectra requires specifically designed measurements with a large dynamic range. With existing techniques, spectra can be recorded over five orders of magnitude using CCD detectors operated in specifically optimized readout mode [18]. In Ref. [19], we presented spectra measured in the Tore Supra tokamak, the far line wings of which were behaving in a way consistent with a power law. However, reliable determination of the exact nature of the these deviations (exponential or power law behavior) would require improved measurements. In fact, the latter spectra were obtained as a by-product of routine measurements, and therefore line wings were recorded only over a limited range. These spectra nevertheless provided clear indications of the presence of an unexpected behavior in the far line wings, and are a strong incentive for further investigations.

\section{CONCLUSIONS AND PERSPECTIVES}

We have shown that as a first approximation the ion temperature field in edge plasmas can be described as a passive scalar, advected by the fluctuating electric drift velocity field. The average temperature gradient naturally provides a forcing term for the fluctuations. The PDF of the passive scalar can be calculated using results obtained in neutral fluids, and have an exponential fall off. The latter translates into an exponential fall off for Doppler line wings. Therefore, passive spectroscopy might provide information on the statistical properties of ion temperature fluctuations, about which very few is known at this time. The effects on Doppler line shapes are observable when the temperature fluctuation PDF deviates significantly from gaussianity, so that their study could either uncover, or rule out such behavior. This work also provides a strong incentive to study the statistical properties of ion temperature 
fluctuations by direct numerical simulation. This would allow to compare the PDF obtained numerically to those provided by our simplified model.

\section{Acknowledgments}

This work was supported by a collaboration (LRC DSM 99-14) between the Laboratoire de Physique des Interactions Ioniques et Moléculaires and the Département de Recherches sur la Fusion Contrôlée, CEA Cadarache, and by the Department of Energy grant DE FGO2ER54677.

[1] W. Horton, Rev. Mod. Phys. 71, 735 (1999).

[2] B. Scott, Phys. Plasmas 12, 062314 (2005).

[3] N. Bretz, Rev. Sci. Instrum. 68, 2927 (1997).

[4] T. H. Evensen et al., Nucl. Fusion 38, 237 (1998).

[5] A. Huber et al., Plasma Phys. Control. Fusion, 49, 409, (2005).

[6] Y. Marandet et al., Contrib. Plasma Phys. 44, 283 (2004).

[7] Y. Marandet et al., Europhys. Lett. 69, 531 (2005).

[8] J. D. Hey et al., J. Phys. B: At. Mol. Opt. Phys. 37, 2543 (2004).

[9] H. R. Griem, Principles of Plasma Spectroscopy, (Cambridge University Press, 1997).

[10] S. I. Braginskii, Rev. Plasma Phys. 1, 205 (1965).

[11] B. Scott, Plasma Phys. Control. Fusion, 39, 16351997.

[12] B. I. Schraiman and E. D. Siggia, Phys. Rev. E 49, 2912 (1994).

[13] G. Falkovich et al., Rev. Mod. Phys. 73, 913, (2001).

[14] Y. G. Sinai and V. Yakhot, Phys. Rev. Lett. 63, 1962 (1989).

[15] Y. Kimura and R. H. Kraichnan, Phys. Fluids A 5, 2264 (1993).

[16] R. T. Pierrehumbert, Chaos 10, 61 (2000).

[17] G. Falkovich et al., Phys. Rev. E 54, 4896 (1996).

[18] J. V. Sweedler et al., Charge Transfer Devices in spectroscopy, (VCH, 1994).

[19] Y. Marandet et al., Communications in non linear science and numerical simulations, 8, 469 (2003). 